\title{
STRESS RECOVERY DURING EXPOSURE TO NATURAL SOUNDS AND ENVIRONMENTAL NOISE

\author{
Jesper Alvarsson
}

\begin{abstract}
Research suggests that physiological stress reactions may be reduced by visual impressions from natural environments as compared to urban or built-up environments. The present experiment tested whether similar effects might be found by auditory stimulation. Forty university students were tested in an experiment with four consecutive recovery sessions after stressful mental arithmetic tests. The independent variables were type of sound during recovery. The sound was either a natural sound environment (sounds from water and birdsong, at $50 \mathrm{dBA}$ ), or three types of noisy environments (traffic noises at 50 or $80 \mathrm{dBA}$ or ambient background sound at $40 \mathrm{dBA}$ ). The main dependent variables were physiological recovery from stress, as measured by decrease in heart rate (HR) and skin conductance level (SCL) after the stressful arithmetic test. The main result was that SCL reduction was faster during nature sounds than during the various noises. For HR, no systematic effects of experimental sounds were found. The result for SCL lends some support the hypothesis that exposure to natural sounds facilitate physiological stress recovery.
\end{abstract}

Edward Wilson postulated (1984) in his book Biophilia that humans are genetically programmed with a preference for nature and relative dislike for urban environments, formed from the millions of years of adaptation. He called this thesis biophilia. A concept originally postulated by Erich Fromm as the love for humanity and nature (1964). A year before Biophilia, Roger Ulrich (1983) published his ground breaking article in Science on improved surgical recovery for patients whose windows faced a park rather then a brick wall. This study showed that nature has a positive effect on human health. Since then, many social scientists have put their effort into discovering positive effects of nature on humans in various environments (e.g. Hartig, Kaiser \& Bowler 2001; Maller, Townsend, Pryor, Brown \& Leger, 2005; Parsons, Tassinary, Ulrich, Hebl \& Grossman, 1998; Ulrich et al., 1991). The problems investigated are primarily concerned with urbanization and its effect on, stress, social well-being, and emotion. The findings in research of preference concerning nature and urbanization, points exclusively to the positive effect of green areas and negative effect of urban ones. This shows that design of the public urban living space is important for general health and well being (van den Berg, Hartig \& Staats, 2007). The positive experiences of nature in these studies may of course be a result of inherited perception patterns of surroundings, but it can just as well be the result of attitudes in favor of nature and dislike of urbanity, taught from toddlerhood and throughout the upbringing..

Some researchers have taken a holistic hold on the issue (Frumkin 2001; Kaplan, Kaplan \& Ryan 1998) and tried to describe the overall experience of a human in nature. Methodologically, this is very complex task given that many variables may influence the experience. Accounting for everything is not a feasible scientific undertaking. 
Neither is the question of where the positive experience is coming from. Attempts have been made with visual stimulation and physiological measures to investigate how basic these experiences are. Parsons et al. (1998) used video and sound stimuli to investigate whether stress recovery was related to roadside environment, urban or countryside. Where they found that recovery during nature stimulation was faster then during other conditions.

Self report as a way to measure variables is problematic in many ways. Studies have shown that affect intensity influences the accuracy with which participants can report their arousal. Instead of using self report as a measure of stress it might be more profitable to measure it physiologically (Blascovich \& Kelsey, 1990). Physiological effects during sleep are evidence to the importance of non-conscious measurement. Haralabidis et al. (2008) found that noise exposure during sleep elevated the subjects' blood pressure (BP). Similar negative effects of noise during sleep on heart rate (HR) have also been found (Griefahn, Bröde, Marks \& Basner, 2007). In awake conditions, Lusk, Hagerty, Gillespie \& Ziemba (2004) have shown that BP and HR were positively correlated with noise level (dBA) for industrial labourers, showing the undesirable effects of noise on cardiovascular health during work. This indicates that the negative effects of noise are stable in different levels of consciousness on group level.

The autonomic nervous system can be divided into two functional parts, the sympathic and the parasympathic, the former mobilizes the body for action and the later rest and recovery. Separating these in physiological measuring would yield additional insights into the process behind stress and arousal. Skin conductance level (SCL) is a pure measure of sympathic activity through sweating, HR is predominantly controlled by the parasympathic vagus nerve and influenced by sympathic activity (Blascovich \& Kelsey, 1990).

Ulrich et al. (1991) was some of the first researchers to use physiological measures to investigate nature's effects on stress recovery. They found that faster physiological recovery during exposure to nature compared to urban environments might be related to parasympathic activity. Fredrickson, Mancuso, Branigan \& Tugade (2000) used a mild stress test in form of a fake speech preparation task and then afterwards showed videos inducing emotion. They concluded that the effect of positive recovery stimuli might not be a simple replacement of negative affections with positive, but rather an undoing effect of the cardiovascular reactions to negative emotions.

Noise may also have a positive effect on performance. As the cardiovascular system becomes activated so does the cognitive system, to a certain degree. Studies have shown that during low mental load tasks, noise can have a positive effect on performance (Stansfeld et al, 2005), especially for noise sensitive individuals (Weinstein, 1978). For these persons performance during noise exposure is lowered (Pawlacyk-Luszczynska, Dudarewicz, Waszkowska, Szymczak \& Sliwnska-Kowlaska, 2005; Sandrock, Schütte, \& Griefahn, 2008; Waye et al, 2001), indicating the importance to control for noise sensitivity. Previous research has shown that sympathic activity is influenced by the mental load during relaxation (Wegner, Broome \& Blumberg, 1997), which shows that increased SCL is sometimes indicative of mental load. 
A major challenge is to connect the long-term effects of noise and health (Babisch, 2008), with the many short-term experimental effects shown in various studies (Chiovenda, et al., 2007; Westman \& Walters, 1981; Persson Waye et.al., 2001). Ising and Michalak (2004) showed that there is no immediate connection between the two. One main concern is the focus in mainstream psychology on group comparisons rather than investigation of underlying processes (Bergman, von Eye \& Magnusson, 2006; Molenaar, 2004). To assess this problem, a more advanced mathematical framework needs to be applied especially since many areas of psychology are heading into research focused on biological processes that interact with and create various psychological phenomena.

A problem concerning validity is also the fact that the choice of sound stimuli is as important as the principles for measurement. A commonly used term in environmental psychology is that of noise, but as Berglund and Nilsson have shown (2003) noise can be separated into different sources (traffic, aircraft, train), which gives different thresholds for identification. It has been shown that unfamiliarity of accent increases mental load in participants (Adank, Evans, Stuart-Mill \& Scott, 2009). Both these studies indicates that identification of the sound source is involved in the sound processing and therefore also possibly the physiological response, indicating that generalization might be constrained in a limited stimuli sample.

The main purpose of this study was to explore whether sound stimulation from different environmental sources can influence physiological recovery from stress. Both HR and SCL were used as indicators of physiological stress in order to obtain measures related to parasympathic and sympathic activity. The hypothesis was that listening to noise while resting after stress increases the time needed to reach physiological relaxation, in comparison to listening to naturalistic and ambient sounds. Another aim was to see whether there where any effects on subsequent performance after sound stimulation.

\section{Method}

\section{Participants}

Forty university students participated in the experiment (24 women and 18 men, mean age $=27$ years). As compensation they could either chose course credit or a payment of $\sim 13$ \$ (100 SEK). HR data was missing from three participants (1 man, 2 woman) due to electrode failure.

\section{Experimental design}

The experiment consisted of three types of exposure: (1) A quiet baseline period, five minutes, (2) periods of testing ("stressor"), two minutes each and (3) periods of relaxation, four minutes each, during which various types of sounds were presented. Each participant was exposed to four different sounds during relaxation. Figure 1 illustrates the experiment schematically. Total time for the experiment was around 45 minutes. 


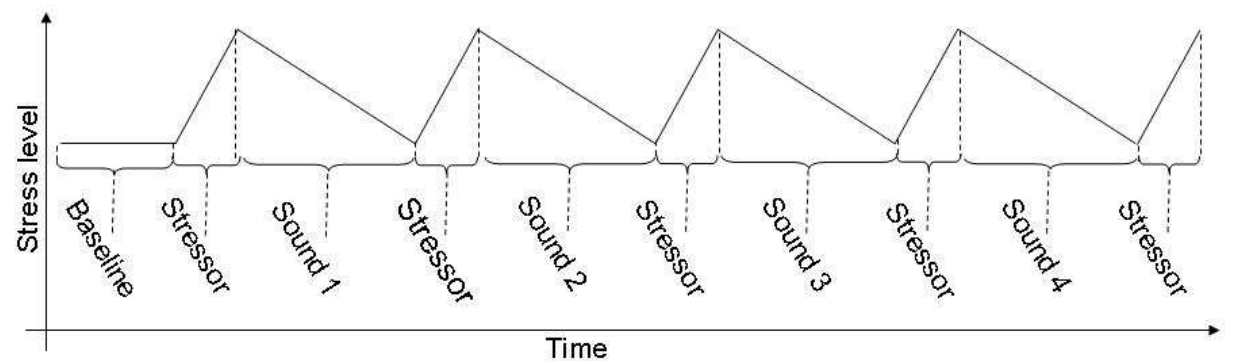

Figure 1. Experimental design with duration and expected stress level on the axis.

A 4 x 4 mixed design was used, with sound during relaxation as within subject variable and presentation order of the four sounds as between subject variable (the sounds are described in detail below). Possible order effects were controlled with a Latin square design, described in Table 1. The participants were randomly assigned to one of the four orders of experimental sounds.

Table 1. 4 x 4 Latin square design of experimental sounds.

\begin{tabular}{cllll}
\hline & \multicolumn{4}{c}{ Experimental groups } \\
\cline { 2 - 5 } $\begin{array}{l}\text { Order of } \\
\text { experimental } \\
\text { sounds }\end{array}$ & Group 1 & Group 2 & Group 3 & Group 4 \\
\hline 1 & Natural & High noise & Ambient & Low noise \\
2 & High noise & Natural & Low Noise & Ambient \\
3 & Ambient & Low noise & Natural & High noise \\
4 & Low noise & Ambient & High noise & Natural \\
\hline
\end{tabular}

\section{Stressor}

The stressor was a two minute speeded mental arithmetic task (referred to as stress test). The task was to decide, within $3 \mathrm{~s}$, whether a displayed equation was correct or false by pressing one of two keys on a numeric keyboard. The responses were evaluated as either "correct", "false" or "too late" (if later than $3 \mathrm{~s}$ ). Feedback was presented on the screen (correct, false or too late) and through earphones with a specific sound for each type of feed-back. The equations consisted of simple arithmetic operations, such as:

$$
543-345=193 \text {, }
$$

The first two terms were integers between 2 and 999, and the answer was a positive whole number below 1000 which either was correct or false (correct answer +/- 3). The operator could either be addition, subtraction, division or multiplication. Each sign had 250 equations in a database, half correct and half false. Overall performance (percent correct) was continuously updated and displayed to the participants in the upper left corner of the screen. 


\section{Experimental sounds}

During each recovery period the participant was exposed to one of four experimental sounds for $4 \mathrm{~min}$. The four sounds were (1) Natural sound, (2) Ambient sound, (3) Low noise sound and (4) High noise sound.

(1) The natural sound was a mixture of sounds from a fountain and tweeting birds. The sound level was set to $50 \mathrm{~dB} L_{\text {Aeq,3min. }}$.

(2) The ambient sound was a recording of a quiet backyard, with a constant low level city hum, considered as a commonly experienced quiet environment. The sound level was set to $40 \mathrm{~dB} L_{\text {Aeq,3min. }}$

(3) The Low noise sound was traffic noise recorded close to a densely trafficked highway. The sound level was set to $50 \mathrm{~dB} L_{\text {Aeq,3min }}$.

(4) The High noise was the same traffic noise as the Low noise, but presented at a considerably higher level, $80 \mathrm{~dB} L_{\text {Aeq,3min }}$.

The natural and traffic sounds were chosen such that the number of events and overall characteristics were as similar as possible. The level of the Natural and the Low noise was set equal $(50 \mathrm{~dB})$ in order to explore the effect of source content at a constant sound level.

\section{Dependent measure}

The mean of second 150-270 of the 300 second baseline was used as an estimate of resting state arousal for HR and SCL respectively

HR was recorded throughout the experiment. Three electrodes were applied by the participant themselves under supervision of the experimenter. The first electrode was positioned five centimeters to the right of the upper sternum and the other two on the left and right side of the stomach, just below the ribcage. It was computed from EKG as the distance between R- waves.

SCL was recorded throughout the experiment. Two electrodes were fitted by the experiment leader to the non dominant hand on hypothenar eminence. The SCL was measured as the change in conductance between the two electrodes at $1000 \mathrm{~Hz}$.

The number of corrects responses during the stress test was used as a measure of performance. A response was defined as correct if the right answer was given within 3 seconds. Late answers were coded as incorrect.

\section{Procedure}

Participants were randomized to one of four groups, which were given different presentation orders of experimental sounds. They were tested individually by the experiment leader. The participants were first asked to wash their hands. They were then seated in a soundproof room and given a written description of the experiment. The electrodes were then fitted to their bodies. Lastly the participant received a pair of 
headphones and a trial of the stress was presented during which data output was checked.

Participants were asked to relax in silence during the five minute baseline. When the baseline period was over a prerecorded female voice said that the first test was to begin with two minutes of the stressor. After the stressor, the female voice instructed the participants to relax and one of the four sound stimuli was presented for four minutes. This was then repeated three more times for each participant, with different sounds in each relaxation period (Figure 1).

At the end of the experiment, the participants answered questions about their age, sex, country of upbringing, number of years in college, number of years studying psychology and three questions measuring noise sensitivity (Weinstein, 1978). Afterwards, participants listened to the four experimental sounds one more time and rated the perceived pleasantness, eventfulness, and familiarity on three bipolar category scales (Axelsson, 2007). Finally, the participants' threshold of hearing was tested, their audiogram was determined using an audiometer (Interacoustics Diagnostic Audiometer AD226, Hughson-Westlake method).

\section{Equipment}

The sounds were recorded with a binaural head and torso simulator Brüel \& Kjær type 4100, with two microphones type 4190 and two pre-amplifiers type 2669, one conditioning amplifier NEXUS Brüel \& Kjær type 2690 A 0S4 and a calibrator Brüel \& Kjær type 4231 plus adapter model 0887. A portable computer Dolch NPAC-Plus P111 with a 6-channel LynxTwo sound card stored the recordings with 24 bit resolution and $48 \mathrm{kHz}$ sampling frequency using Sound Forge 7. Editing and mixing was later done with the same program

In the soundproof room, the signal was fed into a digital filter and D/A-converter Rane RPM 26z, and were then presented through Sennheiser HD 600 headphones. The whole listening system was calibrated using a pink-noise signal, which was measured at the point of the listener's ear. The frequency response of the whole listening system was flat within $2 \mathrm{~dB}, 1 / 3$-octave-band levels, 25-16 $000 \mathrm{~Hz}$.

The physiological data was recorded through a Biopac Systems MP100AT, $1000 \mathrm{~Hz}$. HR was measured with a Biopac ECG100C amplifier and Red $\operatorname{Dot}^{\mathrm{TM}} \mathrm{Ag} / \mathrm{AgCl}$ solid gel electrodes and for SCR a Biopac GSR100C amplifier and EDA isotonic gel electrodes were used.

Both programming and presentation of the mental arithmetic stress task was conducted in Matlab 6.5, and the performance results were saved as a text file. For the physiological data Matlab 6.5 was used for analysis. The recordings were divided into the different parts of the experiment, baseline, stress task (1-5) and recovery (1-4). Data were later imported into SPSS 16 for significance testing. Questionnaire data was analyzed in SPSS 16. 
Results

\section{Group differences}

No group difference was found in noise sensitivity (sum of three items), age, years in college and years studying psychology (one-way ANOVA, $\mathrm{F}(3,36)=.634, \mathrm{p}=.536$ ). This indicates that the group randomization was successful.

\section{Perceptual assessment of experimental sounds}

The ratings of the sounds showed that the Natural sound was the most pleasant sound followed by Low noise and Ambient sound which were similar in pleasantness and the High noise sound which clearly was the least pleasant of the sounds, illustrated in Figure 2. As expected, the ambient sound was perceived as least eventful and least familiar, since it contained no audible sources and was perceived as an undifferentiated background sound.

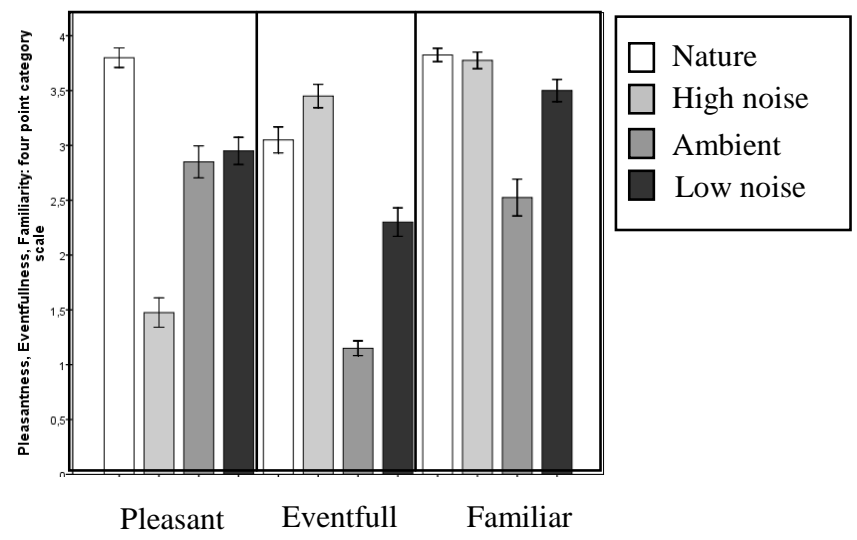

Figure 2: Mean values of pleasantness, eventfulness and familiarity for all sounds. Error bars represents the standard error of the mean

Performance during stress test

To minimize group differences in mathematical skill, results from the first stress, directly following the quiet baseline period, treated as a baseline measure and was used as covariate in the statistical analyses (Rogers \& Hopkins, 1988). A 4x4 mixed ANOVA showed an effect of presentation order related to improved performance over time $(\mathrm{F}(4,144)=2.918, \mathrm{p}=.023)$. No significant group or interaction effect was found. A second $4 \times 4$ mixed ANOVA failed to find any significant effect of performance after sound condition $(\mathrm{F}(3,108)=.489, \mathrm{p}=.690)$. No significant group or interaction effects were found.

\section{Physiological measures}

For the physiological data, a floating mean of 10 seconds was computed for both the HR and the SCL data. Figure 3 (HR) and Figure 4 (SCL) show these values as a function of time, baseline has been subtracted for easier evaluation (not done in any significance tests). The mean of HR and SCL during the recovery period were used as dependent variables in two 4x4 mixed ANCOVAs, with experimental sound as within 
subject variable, presentation order as between-subject variable, and baseline values as covariate for increased power (Rogers, 1988).

\section{Heart rate.}

A general lowering of $\mathrm{HR}$ is seen 30 seconds after the end of the stress test for all stimuli (Figure 3). During relaxation all participants also went below their baseline ( $\mathrm{y}=$ 0 ), the mean difference being in general -5 beats from baseline level.

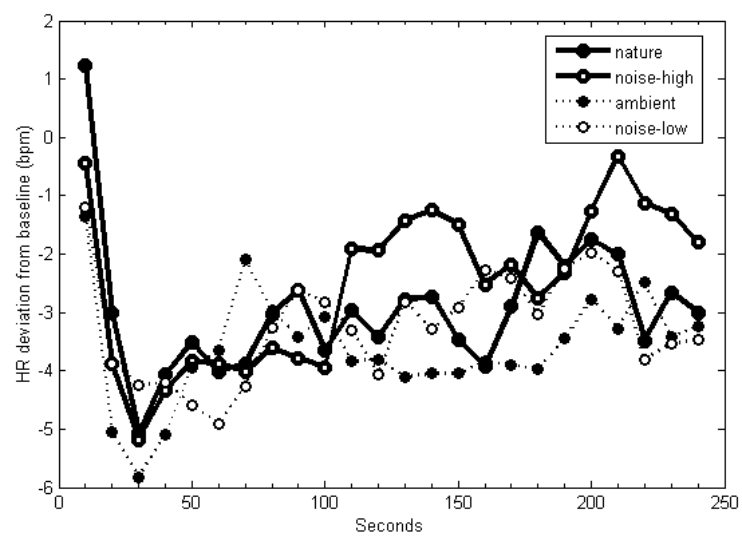

Figure 3. Baseline corrected HR. Floating mean recovery for HR during nature, high noise, ambient and low noise stimuli.

No significant effect of experimental sound was found in HR ANCOVA $(\mathrm{F}(3,96)=.813$ $\mathrm{p}=.490$ ), but a significant interaction effect between experimental sound and presentation order was found $(\mathrm{F}(9,96)=3.973, \mathrm{p}=.001)$. The effect was explained by lower values in HR for Group 1 in the Ambient and Low noise conditions compared to the other groups. No significant between group effect was found.

\section{Skin conductance level.}

For SCL (Figure 4), recovery was faster during the Natural sound than for the other sounds. The Ambient and Low noise sound had the second fastest and High noise the slowest recovery. For unknown reasons, the High noise condition had an upswing during the last 50 seconds of the recovery.

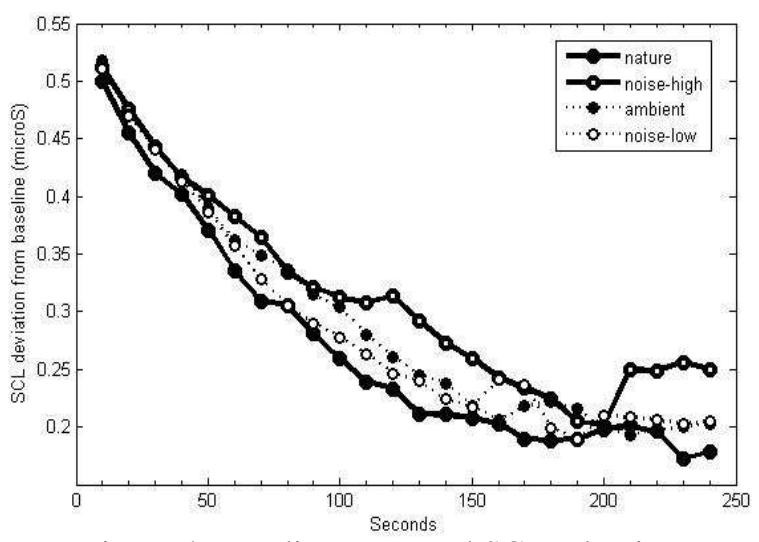

Figure 4. Baseline corrected SCL. Floating mean recovery during nature, high noise, ambient and low noise stimuli. 
The main effect of experimental sound was significant $(F(3,105)=2.731, p=.048)$. Mean SCL was lower for Natural sound than for the other sounds. An interaction effect was also found between group and sound $(\mathrm{F}(9,105)=6.851, \mathrm{p}=.001)$. This was due to order effects where early conditions had relatively lower SCL than later conditions. A pairwise comparison showed a significant difference between natural and high noise $(\mathrm{p}=.045)$, the other paired comparisons were not significant.

To further analyze SCL, a non-linear regression analysis was performed, which fitted an exponential function (Eq. 2) to the average SCL data,

$$
y=b_{1}+b_{2} e^{b_{3} x}
$$

where $y$ is SCL, $x$ is time (in $s$ ) and $b_{1}, b_{2}$ and $b_{3}$ are constants. Figure 5 shows the fitted functions for the four experimental sounds. The fit, $\mathrm{R}^{2}$, for natural, low noise and ambient was $>0.99$, it was slightly lower for noise high, $\mathrm{R}^{2}=0.96$. RMS-error for the Natural, High noise, Ambient and Low noise sound was 0.0088, 0.017, 0.0090 and 0.0097 microS, respectively.

As a measure of the effect of the experimental sound on recovery time, the half life recovery was calculated with the following equation:

$$
x=\frac{\ln \left(\frac{y / 2-b_{1}}{b_{2}}\right)}{b_{3}},
$$

where $\mathrm{y}$ is the intercept when $\mathrm{x}=0$. Each starting point on $\mathrm{y}$ was computed, this corresponds to approximately $.25 \mathrm{~ms}$ for all sounds, which was then divided in two and used as input for the function. The High noise sound had the longest half life of 159.80 $\mathrm{s}$, the half life of the other three were, Ambient $121.31 \mathrm{~s}$, Low noise $111.38 \mathrm{~s}$ and Natural $101.28 \mathrm{~s}$. 


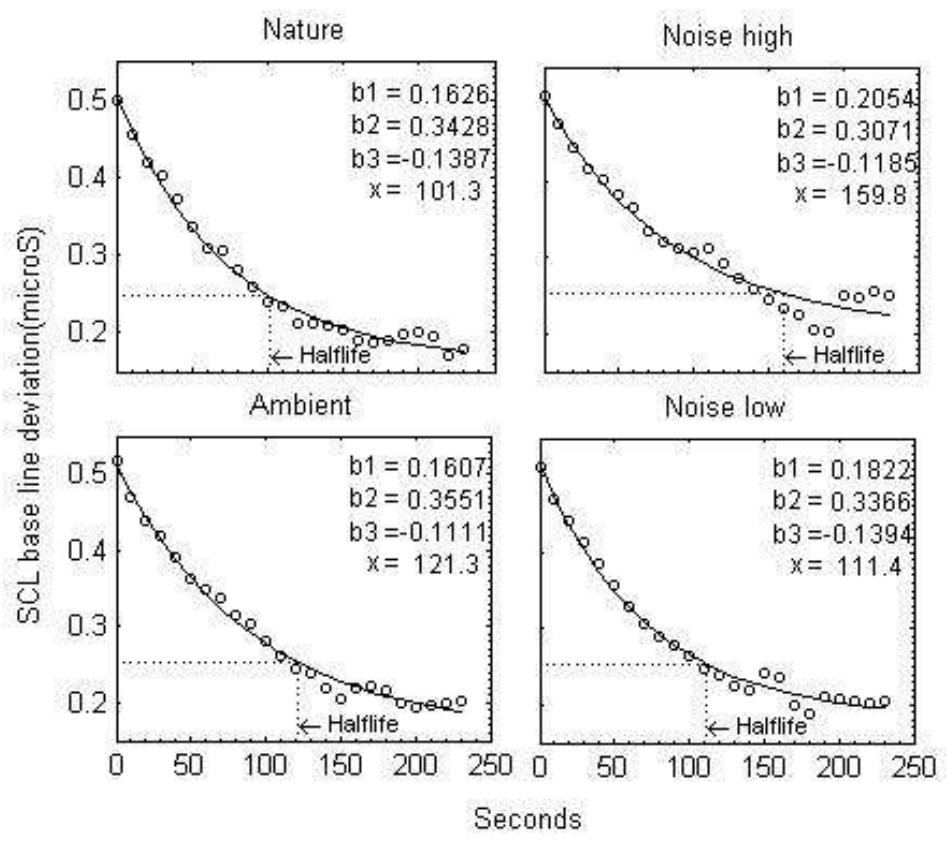

Figure 5: SCL with fitted curves for the group data with constants and halflife value (x) for each sound condition.

Figure 5 refers to group data but there were considerable individual differences, as illustrated by the two individual functions in Figure 6. The left panel shows data from one a participant with a recovery patter similar to the overall group, whereas the right panel shows data from one a participant with a very irregular recovery pattern. For this reason, it was not possible to derive parameter of Equation 2 from every participant and then use these as dependent variables in ANCOVA analyses, as the bad fit resulted in complex numbers due to the natural logarithm.
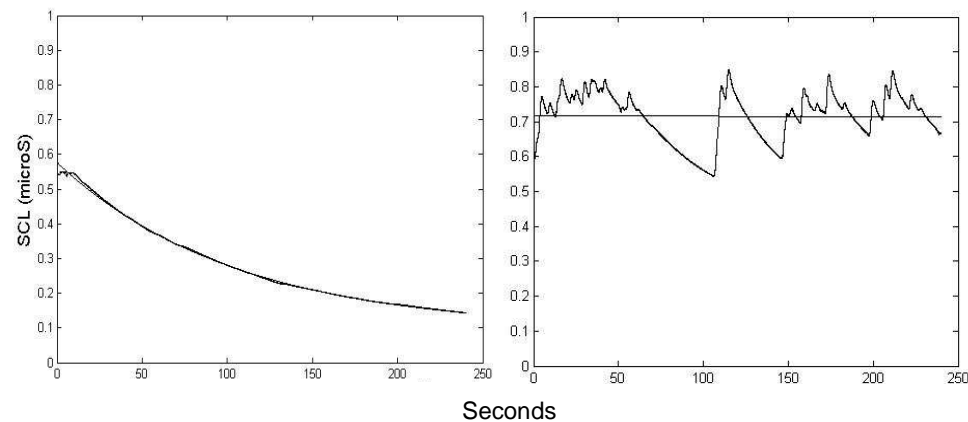

Figure 6: SCL during recovery and curve fit for the nature condition for two participants. 


\section{Discussion}

The main purpose of this study was to test whether physiological stress recovery is faster during exposure to natural sound than to noise. The main result was that SCL reduction was faster during natural sounds than during noise. Half time SCL recovery was 9.09-36.62 percent faster during the Natural sound than during noise. The average SCL after the Natural sound was lower than for the noises, resulting in a significant main effect of experimental sounds in an ANCOVA. For HR, no systematic effects of experimental sounds were found.

SCL is a measure of sympathic activation. The present results suggest that recovery from such arousal is affected by the type of sound during recovery. These results agree with previous findings with combined auditory and visual stimulation (Hartig et al., 2001; Maller et al., 2005; Parsons et al., 1998; Ulrich et al. 1991). They found beneficial effects of natural environments compared to urban environment. These experiments have not specifically looked at the sound environment. Often, sound level and type of sound were confounded. For example, in Ulrich et al. (1991), natural sounds were presented at lower levels than urban noises. The present results suggest that sound level as such seems to be less important than type of sound, because recovery during natural sounds was faster than during noises of lower, equal or higher sound level. The effect of sound type could be related to positive emotions towards nature, as the natural sound was perceived as more pleasant than the noises (Figure 1). As suggested by Fredrickson et al. (2000), positive emotions may undo negative emotions evoked by a stressor. If so, the positive emotion would be the mediator of the effect of nature on recovery.

In the current study, no systematic effects were found for HR. The changes in recovery were much faster then for SCL. In the HR pattern there was a low dip at about 30 seconds that was similar for all sounds, followed by a slowly increasing pattern that seemed to be unrelated to type of background sound (Figure 3). This suggests that HR recovers fast, a process that may be completed before any effects of the sound environment. The lack of consistent results for HR suggests that alternative measures should be used in future studies, for example heart rate variability which may be divided into a sympathic and parasympathic component (Blascovich \& Kelsey, 1990).

The present study used a specific set of experimental sounds, that affect the external validity of the results, as other types of noise and natural sounds might give different results. The Natural sound had a relatively high sound level (50 dBA) in order to be comparable in level to typical urban traffic noise. It may be expected that a natural sound with lower sound level would improve recovery further, since, in general, physiological reactivity is positively related to sound level (Lusk et al., 2004). However, the weaker effect on SCL recovery for the Ambient sound (40 dBA) compared to the Natural sound (50 dBA) shows that type of sound may be more important than sound level. Unlike the Natural sound, the Ambient sound was not possible to identify as specific sound sources. The lack of identification might have caused an increased mental activity and therefore an increased SCL (Wegner et al., 1997).

The results of this experiment supports the view of van den Berg et al. (2007), that urban developers should not focus alone on the visual design but should also consider 
the soundscape as an important part of a positive urban environment. To further advance this area of research, cross modal studies are needed, with high control of stimulus intensity as well as its meaning (e.g., source identification). For practical applications, it is important to determine how modalities interact in forming a positive environment, especially in urban areas. These typically contain both positive and negative components (e.g., pleasant natural sounds and annoying traffic noise). Auditory research may contribute with knowledge on how the auditory system identifies positive stimulation and how this affect is perceived as well as physiological reactions to the overall environment.

\section{Conclusion}

The present results lends some support to the hypothesis that physiological recovery after stress is faster during exposure to natural sounds than during exposure to noise of lower, similar or higher sound pressure levels. 


\section{References}

Adank, P., Evans, B. G., Stuart-Mill, J. \& Scott, S. K. (2009). Comprehension of familiar and unfamiliar native accents under adverse listening conditions. Journal of Experimental Psychology: Human Perception and Performance, 35, 520-529.

Axelsson, Ö. (2007). Individual differences in preferences to Photographs. Psychology of Aestehtics, Creativity, and the Arts, 1, 61-72.

Babisch, W. (2008). Road traffic noise and cardiovascular risk. Noise and health, 10, 27-33.

Berglund, B. \& Nilsson, M. E. (2003). Identification of sounds from traffic. Perceptual and Motor Skills, 97, 675-688

Bergman, L. R., von Eye, A \& Magnusson, D. (2006). Person-Oriented Research Strategies in Developmental Psychopathology. Developmental psychopathology, 1, 850-888.

Blascovich, J. \& Kelsey, R. M. (1990). Using Electrodermal and Cardiovascular Measures of Arousal in Social Psychological Research. Review of Personality and Social Psychology, 11, 45-73.

Chiovenda, P., Pasqualetti, P., Zappasodi, F., Ercolani, M., Milazzo, D., Tomei, G., et al (2007). Environmental noise-exposed workers: Event-related potentials, neuropsychological and mood assessment. International Journal of Psychophysiology, 65, 228-237

Fredrickson, B. L, Mancuso R. A., Branigan, C. \& Tugade, M. M. (2000). The Undoing Effect of positive Emotions. Motivation and Emotion, 24, 237-258

Fromm, E. (1964). The Heart of Man. New York: Harper \& Row.

Frumkin, H. (2001). Beyond Toxicity: Human Health and Enviroment. American Journal of Preventive Medicine. 20, 234-240

Harilabidis, A., Dimakopoulou, K., Vigna-Taglianti, F., Giampaolo, M., Borgini, A., Dudley, M. L., et al. (2008). Acute effects of night-time noise exposure on blood pressure in populations living near airports. European Heart Journal, 29, 658-664.

Hartig, T., Kaiser, F. G., \& Bowler, P. A. (2001). Psychological restoration in nature as a positive motivation for ecological behaviour. Environment and Behaviour, 33, 590-607.

Griefahn, B., Bröde, P., Marks, A. \& Basner, M. (2007). Autonomic arousals related to traffic noise during sleep. Sleep, 31, 569-577.

Ising, H. \& Michalak, R. (2004). Stress effects of noise in field experiment in comparison to reaction to short term noise exposure in the laboratory. Noise and health,59, 392-399.

Kaplan, R., Kaplan, S., \& Ryan, R. L. (1998). The experience of nature: A psychological perspective. New York, NY: Cambridge University Press

Lusk, S., Hagerty, B., Gillespie, B. \& Ziemba, R. (2004). Acute effects of noise on blood pressure and heart rate. Archives of Environmental Health, 59, 392-399.

Maller, C., Townsend, M., Pryor, A., Brown, P. \& Leger, L. (2005). Healthy nature healthy people: 'contact with nature' as an upstream health promotion intervention for populations. Oxford University Press, 21, 45-54.

Molenaar, P. C. M. (2004). A manifesto on psychology as an idiographic science: Bring the person back into scientific psychology: This time forever. Measurement: interdisciplinary Research and Perspectives, 2, 201-218

Parsons, R., Tassinary, L. G., Ulrich, R. S., Hebl, M. R. \& Grossman A. M. (1998). The view from the road: Implications for stress recovery and immunization. Journal of Environmental Psychology, 18, 113-139.

Pawlacyk-Luszczynska, M., Dudarewicz, A., Waszkowska, M., Szymczak, W. \& Sliwnska-Kowlaska, M. (2005). The impact of low frequency noise on human mental performance. International Journal of Occupational Medicine and Environmental Health. 18, 185-198. 
Rogers, W. T. \& Hopkins, K. (1988). Power estimates in the presence of covariate and measurement error. Educational and psychological measurement. 48, 647-656.

Sandrock, S., Schütte, M., \& Griefahn, B. (2008). Impairing effects of noise in high and low noise sensitive persons working on different mental tasks. International Archives Occupational Environmental Health, 82, 779-85.

Stansfeld, S., Berglund, B., Clark, C., Lopez-Barrio. I., Fischer, P., Öhrström, E. \& Haines, M. M. (2005). Aircraft and road traffic noise and children's cognition and health. A cross-national study. Lancet, 365, 1942-1949.

Ulrich, R. S. (1983). View Through a Window May Influence Recovery from Surgery. Science, 224, 420-421.

Ulrich, R. S., Simons, R. F., Losito, B. D., Fiorito, E., Miles, M. A. \& Zelson, M. (1991). Stress recovery during exposure to natural and urban environments. Journal of Environmental Psychology, 11, 201-230.

van den Berg, A., Hartig, T. \& Staats, H. (2007). Preference for nature in urbanized societies: stress, restoration, and the pursuit of sustainability. Journal of Social Issues, 63, 79-96.

Waye, K. Persson, Bengtsson, J., Rylander, R. Hucklebridge, F., Evans, P. \& Clow, A. (2001). Low frequency noise enhances cortisol amoing noise sensitive subjects during work performance. Life Sciences, 70, 745-758.

Wegner, D. M., Broome, A. \& Blumberg, S. J. (1997). Ironic effects of trying to relax under stress. Behavior Research and Therapy, 35, 11-21.

Weinstein, N. D. (1978). Individual differences in reactions to noise: A longitudinal study in a college dormitory. Journal of Applied Psychology, 63, 458-466.

Westman J. C. \& Walters J. R. (1981). Noise and stress: a comprehensive approach. Environ Health Perspectives 41, 291-309

Wilson, E. O. (1984). Biophilia. Cambridge: Harvard University Press. 